\title{
POLYCYSTIC OVARY SYNDROME
}

\author{
Swarup S Patel, MRCOG; Vincent Bamigboye, MRCOG, MSc
}

The term polycystic ovary syndrome (PCOS) is a misnomer as it gives an impression of an ovarian disease with cysts. It is, however, a multisystem disorder with very distressing signs and symptoms and long term consequences on the well being of the patient. Mr Patel and Mr Bamigboye, respectively specialist registrar and consultant in obstetrics and gynaecology, describe this common and complicated condition and its modern management.

\section{INTRODUCTION}

PCOS is a common condition affecting $5-10 \%$ of women of reproductive age. Twenty per cent of white women of reproductive age in the United Kingdom (UK) show features of polycystic ovaries on ultrasound. The prevalence of PCOS is higher in some ethnic groups - South Asian immigrants to UK. PCOS shows a familial clustering being more common in women with affected first degree relatives. Hyperandrogenaemia appears to be the strongest genetically inherited characteristic in familial cases. It is a dominantly inherited trait with low penetrance and variable expressivity.

Our understanding of the condition has evolved immensely since the condition was first described in 1935 by Irwin F Stein and Michael L Leventhal, as Stein-Leventhal Syndrome. They described seven patients with amenorrhoea, hirsutism and enlarged polycystic ovaries; four of whom were obese. After resection of half to three quarters of the ovaries all of them resumed regular menses and two of them became pregnant.

\section{DEFINITION}

The term PCOS has been used variously by different individuals. However, an international consensus regarding the definition of PCOS was reached at the joint meeting of the American Society of Reproductive Medicine and the European Society of Human Reproduction and Embryology. Two of the following three criteria should be present to define a condition as PCOS:

- oligomenorrhoea or amenorrhoea and/or anovulation

- clinical or biochemical hyperandrogenism

- polycystic ovaries

A polycystic ovary is defined as presence of 12 or more follicles of $2-9 \mathrm{~mm}$ in diameter or an ovarian volume of more than $10 \mathrm{~cm}^{3}$. Typically, there is increased stroma with multiple peripherally arranged follicles. Polycystic ovaries on ultrasound need not be present to make a diagnosis of PCOS and the presence of polycystic ovaries does not establish the diagnosis of PCOS

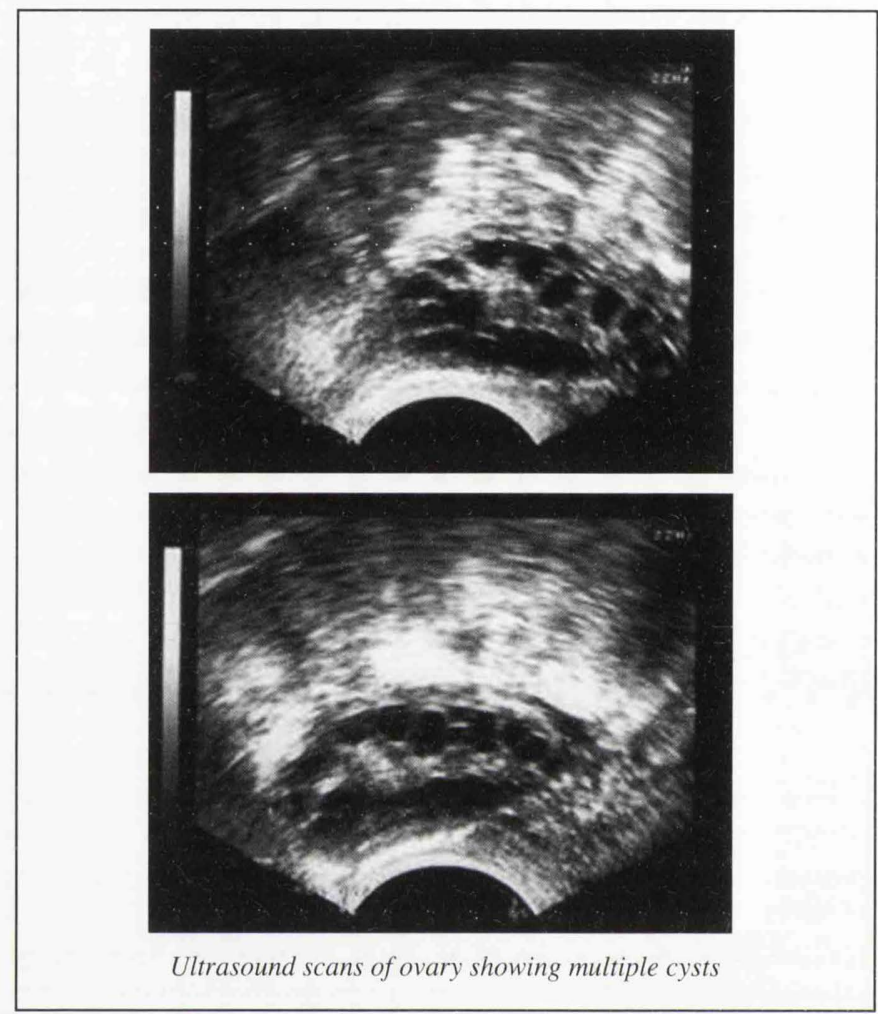

PCOS is diagnosed after exclusion of other medical conditions. The following conditions need to be ruled out to establish the diagnosis of PCOS:

1. Non-classic congenital adrenal hyperplasia due to deficiency of 21-hydroxylase.

2. Cushing's syndrome.

3. Primary hypothyroidism.

4. Hyperprolactinaemia/prolactinoma.

5. Acromegaly.

6. Premature ovarian failure.

7. Simple obesity.

8. Androgen-secreting adrenal or ovarian neoplasm.

9. Intake of drugs such as androgens, valproic acid or cyclosporine.

\section{PATHOPHYSIOLOGY}

PCOS is polygenic and multifactorial. There is inherent ovarian dysfunction which is influenced strongly by extraovarian factors - disturbances in the hypothalmo-pituitaryovarian axis and hyperinsulinaemia. There is increased luteinising hormone ( $\mathrm{LH})$ pulse frequency in women with PCOS which may be due to increased pulse frequency of gonadotrophin-releasing hormone $(\mathrm{GnRH})$. Increased pulse 
frequency of GnRH may be either due to intrinsic abnormality in the GnRH pulse generator or to relatively low levels of progesterone. Oestrone is elevated in PCOS and may play a role in LH hypersecretion. Increased Inhibin-B secretion by the polycystic ovaries causes inhibition of follicular stimulating hormone (FSH) and an increase in the $\mathrm{LH} / \mathrm{FSH}$ ratio. $\mathrm{LH}$ excess causes ovarian hyperandrogenism by stimulating the theca cells of the ovary.

Insulin resistance is seen in PCOS: this is due to postbinding abnormality ${ }^{(1)}$, and leads to compensatory hyperinsulinaemia. Insulin enhances theca cell androgen production by binding to insulin-like growth factor 1 (IGF-1) receptor. Hyperinsulinaemia also decreases the sex hormone binding globulin (SHBG) production by the liver and thus increases the free androgen levels and consequent peripheral androgen action. Androgens also decrease the SHBG levels thus causing a vicious cycle. Hyperinsulinaemia also inhibits IGF binding protein-1 which further augments ovarian androgen production $^{(2)}$. Insulin also increases endogenous androgen production by increasing cytochrome enzyme activity.

Intraovarian androgen excess promotes follicular atresia and increased stromal compartment which retains LH responsiveness and continues to secrete androgens.

\section{SYMPTOMS}

\section{Obesity}

Thirty-five to fifty per cent of women with PCOS are overweight or obese. These women typically have central obesity with increase in waist/hip ratio. Obesity tends to worsen anovulation through increased peripheral oestrogen production. Obesity enhances the features of insulin resistance and is associated with menstrual irregularity, infertility and complications during pregnancy and childbirth. Abdominal obesity also increases serum androgens and LH. Increased insulin levels interfere with the hypothalamicpituitary-ovarian axis by increased production of $\mathrm{LH}$ which interferes with follicular maturation. Weight loss can improve the menstrual cycle infertility, restore ovulation and normalise biochemical indices - insulin resistance ${ }^{(3)}$. Weight loss also decreases hirsutism, free androgen excess, free and total androgens and increases $\mathrm{SHBG}^{(4)}$. Obesity increases the risk of cardiovascular disease and diabetes mellitus in the aging PCOS women.

\section{Menstrual disturbances}

The majority of women with PCOS have menstrual disturbance - infrequent menstruation associated with anovulation. Approximately $30 \%$ have normal regular menstrual cycles, $50 \%$ have oligomenorrhoea and $20 \%$ have amenorrhoea. Eighty-five to ninety per cent of women with oligomenorrhoea and $30-40 \%$ of women with amenorrhoea will have PCOS.

\section{Reproductive dysfunction}

PCOS is associated with anovulation. PCOS is diagnosed in $75 \%$ of women with anovulatory infertility. Polycystic ovaries have increased number of primary and secondary follicles which are viable and involved in steroidogenesis and are capable of responding to gonadotrophins. There is absence of a dominant follicle and follicular growth is arrested. The exact cause for this is unknown but increased $\mathrm{LH}$, hyperandrogenaemia and hyperinsulinaemia have been implicated. Relative FSH deficiency has also been implicated in the failure of follicular development. This may be due to increased ovarian steroid concentration resulting in negative feed back to the hypothalmo-pituitary axis or due to increased inhibin production. Androgens enhance aromatase activity and inhibin production. Insulin acts synergistically with $\mathrm{LH}$ in stimulating androgen production in the theca cells. Hyperandrogenism is seen in both ovulatory and anovulatory women with polycystic ovaries. Hyperinsulinaemia induces premature maturation of the granulosa cells and arrests follicular maturation.

Growth differentiation factor 9 (GDF-9), epidermal growth factor (EGF) and transforming growth factor (TGF- $\alpha$ ) and the cytokine, tumour necrosis factor (TNF- $\alpha$ ) have been implicated in the pathogenesis. Other contributory factors include decreased plasminogen factor inhibitor activity, vascular endothelial growth factor (VEGF) and obesity.

Women with PCOS who conceive either spontaneously or with treatment have a higher incidence of miscarriage compared to the general population. Increased LH levels have been implicated in early pregnancy loss ${ }^{(5)}$. Other related factors which may contribute to the early pregnancy loss are hyperandrogenaemia, insulin resistance, abnormal folliculogenesis, obesity and the fertility treatment itself. Abnormalities in plasminogen activator inhibitor (PAI) activity, abnormal vascular reactivity secondary to endothelial dysfunction and abnormal endometrial receptivity are other possible explanations for the increased risk of miscarriages

\section{Acne}

Both acne and hirsutism are common clinical symptoms in women with PCOS. Obesity, acne and hirsutism are responsible for considerable psychological distress and negative body image in these women who may be trying to cope with decreased fertility.

Acne is commonly seen on the face. However, $50 \%$ of hyperandrogenic women have acne on the neck, chest and upper back. It is due to a combination of sebum, follicular epithelial cells and propionibacterium acne which results in the papules, pustules and nodules of acne.

Androgens increase sebaceous secretion which is a precipitating factor for acne formation. Androgens also cause abnormal follicular epithelial desquamation that is required for the formation of comedone. Acne formation is associated with androstenedione, dehydroepiandrosterone (DHEA) and dehydroepiandrosterone sulphate (DHEAS) but not dihydrotestosterone (DHT).

\section{Hirsutism}

Hirsutism is defined as excessive facial and/or body terminal hair in a male-like distribution. There are three types of hair: lanugo is present at birth and is lost in the immediate postpartum period; vellus hair is non-pigmented, soft and short; terminal hair is pigmented, coarse and longer than vellus hair. Testosterone and DHT transform vellus hair into terminal hair in areas of the body which are androgen sensitive. The conversion which occurs over several growth cycles is irreversible. Abnormal elevation of serum androgens stimulate excessive hair growth on face, neck, chest and lower abdomen. The rate of hair growth varies due to genetic differences in the activity of $5 \alpha$-reductase, which converts testosterone to the more potent metabolite, DHT. Enzymatic activity of $5 \alpha$-reductase is stimulated by hyperandrogenism, insulin and IGF.

Seventy per cent of women with hirsutism have PCOS The differential diagnosis of hirsutism includes hyperthecosis, non-classic adrenal hyperplasia (NCAH), 


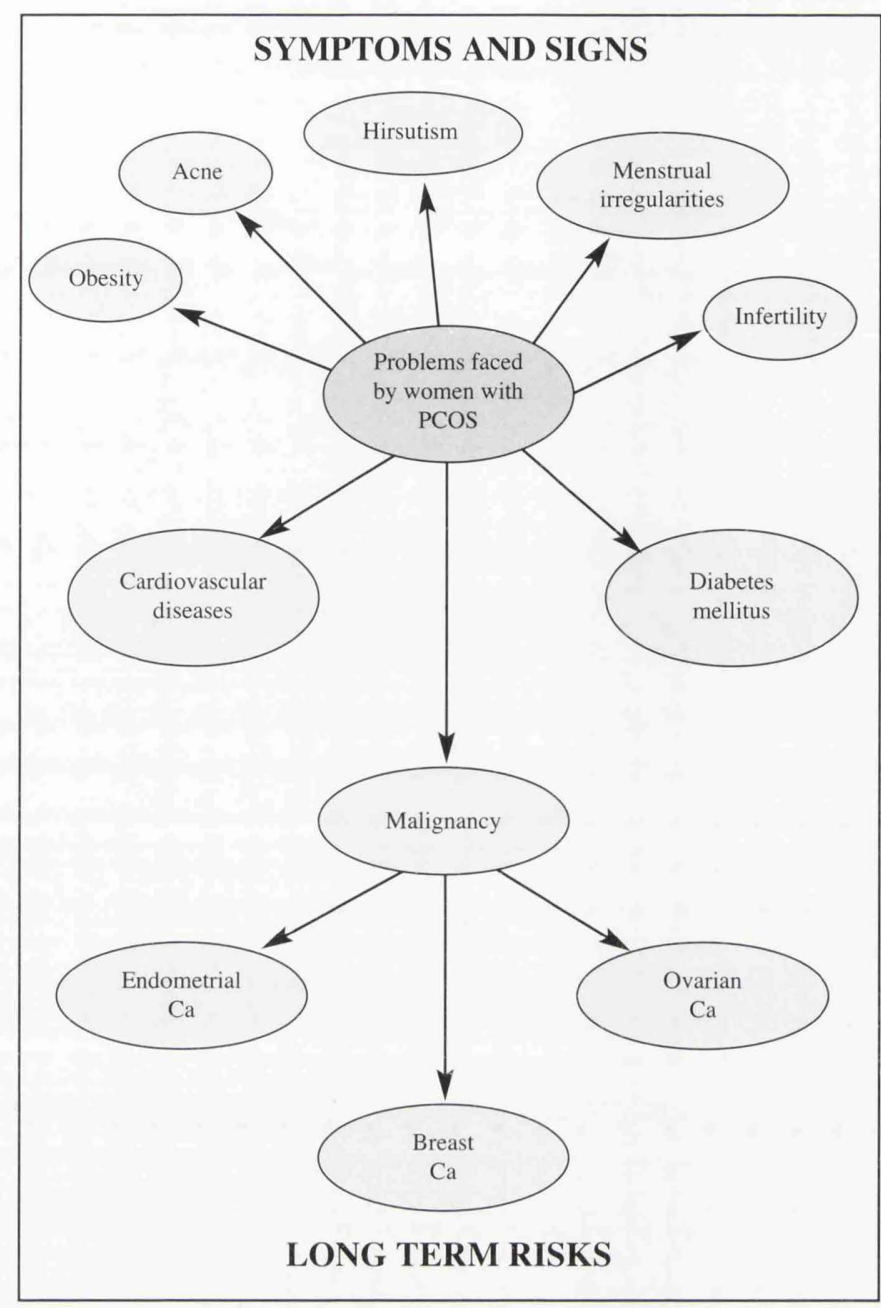

Cushing's syndrome, thyroid dysfunction and ovarian and adrenal androgen producing tumours ${ }^{(6)}$. The prevalence of androgen-secreting tumours in a hirsute patient is less than $0.5 \%$.

\section{Androgenic alopecia}

This is described as progressive loss of scalp terminal hair leading to baldness. Sixty-seven per cent of women with androgenic alopecia have $\operatorname{PCOS}^{(7)}$. Twenty-one per cent of women with androgenic alopecia are hirsute. For androgenic alopecia to develop a familial predisposition to baldness along with hyperandrogenism is required.

\section{Acanthosis nigricans}

This is a mucocutaneous eruption manifest by increased pigmentation and papilomatosis. It commonly occurs in the axillae, skin flexures and nape of the neck. It is a marker of insulin resistance and increased insulin secretion ${ }^{(8)}$. One to three per cent of women with PCOS show features of acanthosis nigricans. It is more common in adolescents.

\section{METABOLIC ABNORMALITIES AND INSULIN RESISTANCE}

The pathogenesis of PCOS is complex and not yet fully understood. Insulin resistance has been proposed as the key metabolic defect in its aetiology. Insulin resistance is defined as inability of insulin to perform its physiological actions reduction in its ability to lower blood glucose.

Obesity (abdominal obesity) has been implicated in insulin resistance in patients with or without PCOS probably through the release of free fatty acids (FFA) from adipocytes. There is impaired uptake of glucose in skeletal muscles, adipocytes and liver and increased hepatic glucose output ${ }^{(9)}$. Insulin resistance leads to compensatory hyperinsulinaemia.

Hyperandrogenaemia correlates with insulin resistance in both obese and lean women with PCOS. The ovarian tissue does not appear to have insulin resistance unlike the skeletal and adipose tissue. Insulin synergistically increases the action of LH, stimulating androgen production in the theca cells. In the liver, insulin decreases the production of SHBG, thus increasing the total and free androgen levels.

Not all women with PCOS exhibit hyperinsulinaemia and insulin resistance ${ }^{(10)}$. This may be due to variable criteria applied for diagnosis of PCOS in the earlier studies or differing methods used for measuring insulin sensitivity. Ratio of fasting glucose/insulin, insulin response to oralglucose tolerance test (OGTT) or OGTT glucose/insulin ratio are more sensitive in measuring insulin resistance than fasting insulin levels alone.

\section{LONG TERM RISKS}

\section{Cardiovascular disease}

Coronary artery calcification is seen more commonly in PCOS than in controls, particularly in the obese group ${ }^{(11)}$. Decreased antioxidant capacity seen in PCOS may be exaggerated by insulin resistance. Reduced fibrinolysis and platelet dysfunction have been demonstrated in women with PCOS. There is a negative correlation between platelet aggregability and insulin sensitivity ${ }^{(12)}$.

Women with PCOS have lower high density lipoproteins (HDL) levels, higher triglyceride levels and higher low density lipoprotein (LDL)/HDL ratio. Both insulin and body fat distribution play an important role in regulating lipid levels.

Young women with PCOS have a normal blood pressure but fail to show the normal fall in blood pressure at night. At menopause, women with PCOS have a relative risk of 2.5 for hypertension compared to age-matched controls.

\section{Diabetes mellitus}

Women with PCOS have 5-10 times higher risk of developing type 2 diabetes mellitus ${ }^{(13)}$. Onset of diabetes is earlier - third or fourth decade. Impaired glucose tolerance is seen in $30 \%$ of women with PCOS. Hyperinsulinaemia in PCOS is independent of obesity. In overweight women with PCOS, weight reduction reduces the fasting insulin levels.

\section{PCOS and cancer}

\section{Endometrial carcinoma}

It is widely accepted that unopposed oestrogen is a risk factor for endometrial carcinoma. In PCOS there is chronic anovulation with normal or high levels of oestrogen. It is estimated that women with PCOS have three times increased risk of developing endometrial carcinoma compared to the general population. However, there is a paucity of concrete evidence. There is a reduction of risk in women with PCOS who use oral contraceptives or progesterone.

\section{Breast carcinoma}

Oestradiol has been implicated in development of breast carcinoma. It is more common in women than men. It stimulates breast cancer cell lines in culture and duct breast tissue. Hormone replacement therapy is associated with increased risk of breast carcinoma. Oophorectomy is a 
palliative for some of the breast cancers. PCOS may be postulated to increase the risk of breast carcinoma; however, data available do not demonstrate an increased risk. The explanation may lie in the low progesterone levels in these patients. Further studies need to be performed to demonstrate any association.

\section{Ovarian carcinoma}

Incessant stimulation of ovaries due to high gonadotrophin levels may lead to increased risk of ovarian carcinoma. Also, patients with PCOS are exposed to ovulation induction agents for treatment of their subfertility which can predispose them to ovarian malignancy. However, data available show conflicting results. There is obviously a need to have more robust studies in this area.

\section{MANAGEMENT}

This involves treatment of symptoms and prevention of long term complications.

\section{Weight loss}

In non-PCOS subjects, weight loss and exercise reduce insulin resistance, plasma lipids and blood pressure. In PCOS patients, weight loss and reduction in abdominal fat reduces insulin resistance, circulating insulin levels, hyperlipidaemia, free androgen excess, free and total testosterone and SHBG.

Following weight loss the metabolic and endocrine variables are improved to the level of Body Mass Index (BMI) matched non-PCOS subjects ${ }^{(14)}$. The reproductive function returns with moderate weight loss $(<10 \%$ of initial body weight) though the BMI may remain more than $30 \mathrm{~kg} / \mathrm{m}^{2}$. Endocrine improvements occurred maximally during energy restriction.

Strategies to reduce energy consumption are beneficial in improving reproductive function. Behavioural modification, physical exercise and high fibre diet are beneficial. Moderate increase in dietary protein, at the expense of dietary carbohydrates, might improve weight loss and improve insulin sensitivity ${ }^{(15)}$.

Insulin sensitising agents can be used but lifestyle modifications are preferable because of potential costs, side effect and need for long term maintenance.

\section{Hirsutism and acne}

Weight reduction may be beneficial in managing these conditions because of the beneficial endocrinological changes. A combined oral contraceptive pill (COCP) is the next line of treatment of these symptoms, especially in a young lady needing contraception if there is no contraindication to COCP. Formulations with low androgenic progestins may be beneficial. Oestrogen and progesterone suppress gonadotrophin secretion and mid-cycle gonadotrophin surge resulting in decreased ovarian steroidogenesis. Oestrogen also increases the SHBG levels resulting in decreased concentration of free testosterone levels ${ }^{(16)}$.

A topical retinoid (tretinoin or adapeline) is useful for acne. Combining a topical retinoid with a topical antibiotic may help reduce side effects and improve clinical outcome. Topical eflornithine hydrochloride $13.9 \%$ is beneficial in treatment of facial hair but the effect is transient. It has minimal side effects - mostly tingling, burning, erythema and rash.
Mechanical hair removal methods such as plucking, waxing and shaving may be used. Electrolysis or laser treatment can provide permanent hair removal.

Various pharmacological agents have been used for hirsuitism. Gonadotrophin-releasing hormone analogues (GnRHa) with or without oestrogen and progestin replacement can be used. GnRHa are expensive and when used alone lead to anti-oestrogenic side effects which include, vasomotor instability, vaginal dryness, reduced breast size, mood swings and insidious loss of bone mineral density. Spironolactone (antiandrogen and aldosterone antagonist), Flutamide (nonsteroidal antiandrogen) or Finasteride (inhibitor of $5 \alpha$-reductase) can be used. Insulin sensitising agents (metformin, pioglitazone and acarbose) decrease insulin resistance and insulin levels, decrease total and free testosterone levels and increase SHBG. However, the effect of metformin on hirsutism is inconclusive with some studies demonstrating an improvement whereas others have not ${ }^{(17)}$.

\section{Infertility and reproductive failure}

The majority of women with PCOS and infertility have anovulation or oligo-ovulation and have menstrual irregularities. Almost all of these women have typical features of polycystic ovaries. Restoration of ovulation can be obtained by reducing insulin and LH levels or by ovarian stimulation

\section{Weight loss}

Loss of weight reduces insulin and androgen concentration and increases SHBG levels. Loss of body weight of $5-10 \%$ is enough to restore ovulation and pregnancy in $55-100 \%$ within six months ${ }^{(18)}$. It is effective, cheap and devoid of side effects. It should be the first line of management in obese women with PCOS with anovulatory infertility.

\section{Ovarian stimulation}

Polycystic ovaries have at least double the number of small antral follicles compared to normal ovaries and are prone to multiple follicular development. Polycystic ovaries have the same threshold of FSH to initiate a response, but doses slightly above the threshold are prone to develop ovarian hyperstimulation syndrome (OHSS).

\section{ClOMIPHENE CITRATE}

This is the first line treatment for ovarian stimulation in PCOS women. It is an anti-oestrogen and blocks the oestradiol receptors in the hypothalamus. This induces a change of GnRH pulse frequency, release of FSH and consequent follicular development. It is given in a dose of $50 \mathrm{mg} /$ day for five days, beginning from the second to the sixth day of spontaneous or withdrawal bleeding. It can be increased in increments of $50 \mathrm{mg} /$ day until an ovulatory cycle is induced. There is no additional benefit of increasing the dose to more than $150 \mathrm{mg} /$ day. Ovulation is restored in about $80 \%$ of women and pregnancy in 35 $40 \%$. This may be due to increased secretion of $\mathrm{LH}$ or to anti-oestrogenic effects on endometrium and cervical mucus. Intrauterine insemination (IUI) can be performed to overcome the problem. Pelvic ultrasound to assess the follicular development and endometrial thickness will identify those who are not responding and those who have depressed endometrium. It increases the costs but prevents protracted periods of inappropriate therapy.

\section{GONADOTROPHINS}

This is indicated when there is no pregnancy after four to six cycles of chlomiphene therapy in a daily dose of $150 \mathrm{mg}$. FSH is given in incremental dose of 75IU every five to seven days followed by hCG 10,000 units to induce 
ovulation. This results in a pregnancy rate of $34 \%$ and severe OHSS in about $4-5 \%$ of cases. The aim is to develop a single dominant follicle thereby avoiding the complications of OHSS and multiple pregnancies. Chronic low dose regimens and step-down protocols have been used to achieve this as severe OHSS can be a lifethreatening condition.

Methods used to decrease the incidence of severe OHSS, especially during IVF cycles are:

- avoid hCG (treatment cancellation)

- delay hCG (coasting)

- reduce hCG dose

- surrogate hCG (LH agonist)

- prophylactic albumin

- aspiration of all follicles

- elective embryo cryopreservation (avoid pregnancy)

- avoid hCG in luteal phase

\section{Reduction of LH concentrations}

Suppressing LH concentrations along with ovulation induction can prevent premature leutinisation, increase pregnancy rates and lower miscarriage rates.

GONADOTROPHIN-RELEASING HORMONE AGONISTS (GNRHA) GnRHa have a higher affinity for GnRH receptors. They thus initially stimulate the receptors but later continue to occupy the receptor sites and produce down regulation. They are, however, not used routinely as treatment cycles are longer, more cumbersome, more expensive and there is a higher incidence of OHSS and multiple pregnancies.

GONADOTROPHIN-RELEASING HORMONE ANTAGONISTS (GNRH ANTAGONISTS)

GnRH antagonists suppress gonadotrophin release within a few hours, have no flare-up effect and gonadal function resumes without a lag effect following their discontinuation. They are more expensive and have not yet been subjected to rigorous trials and are not used routinely in clinical practice.

LAPAROSCOPIC OVARIAN DRILLING (LOD)

The original treatment of PCOS, proposed by Stein and Leventhal, (ie bilateral wedge resection of ovaries) was highly successful. It was abandoned because of high incidence of pelvic adhesions. LOD is a less invasive procedure. Unipolar coagulating current or laser is used to puncture the ovarian surface in four to ten places to a depth of $4-10 \mathrm{~mm}$. This results in ovulation in about $82 \%$ and a pregnancy in about $63 \%$ of patients. It results in similar pregnancy rates to three to six cycles of gonadotrophin therapy with significant reduction of OHSS, multiple pregnancies and miscarriage rates. If pregnancy is not achieved within two to three months, ovulation induction with gonadotrophins maybe performed with increased success rate. There is a dramatic reduction of LH within two days of surgery.

\section{Reduction of hyperinsulinaemia - metformin}

Metformin in a dose of $1500-2500 \mathrm{mg} /$ day results in a significant reduction in insulin, androgens and $\mathrm{LH}$ concentrations. Ovulation occurs in $78-96 \%$ of women with PCOS. Metformin can be used alone or along with clomiphene citrate or gonadotrophins. Metformin when continued in pregnancy appears to be safe and reduces miscarriage rates. The role of metformin in in-vitro fertilisation (IVF) cycles needs further investigation. Its role in IVF/ICSI cycles is controversial with some reporting a benefit ${ }^{(19)}$ whereas others have not been able to demonstrate any advantage ${ }^{(20)}$.

\section{In-vitro fertilisation and embryo transfer}

This is the last resort for women with PCOS where all other options have failed. Fertilisation of recovered eggs is less in women with PCOS but this is balanced by the larger number of eggs recovered. Pregnancy rates following IVF and embryo transfer in PCOS is similar to that of tubal factor infertility. In-vitro maturation (IVM) of oocytes is an option which needs further studies.

\section{PREVENTION OF LONG TERM RISKS}

\section{Diabetes and cardiovascular risks}

A healthy lifestyle and weight reduction should be encouraged to prevent cardiovascular problems. Physical activity must be increased and maintained to minimise the risks. Weight loss is associated with decrease in visceral fat and improved insulin sensitivity and blood pressure even during the early stages of weight reduction. Early identification of glucose intolerance and prevention of progression to type 2 diabetes mellitus may reduce the incidence and severity of atherosclerosis. Type 2 diabetes mellitus can be prevented in middle-aged obese women with PCOS by diet and lifestyle modification. Insulin sensitisers may have a role in prevention of both type 2 diabetes mellitus and cardiovascular diseases in women with PCOS.

\section{Endometrial carcinoma}

Weight reduction may help prevent endometrial carcinoma in PCOS women. Women with PCOS and amenorrhoea should be advised to take progestogens at least every three months or to use the oral contraceptive pill.

\section{CONCLUSION}

PCOS is a complex disease with variable presentation. Carefully documented signs and symptoms and endocrinology need to be considered in making the diagnosis. Early management is necessary to treat the distressing symptoms and signs and also prevent long term consequences.

\section{REFERENCES}

1. Dunaif A. Insulin resistance and the polycystic ovary syndrome: mechanisms and implication for pathogenesis Endocr Rev 1997; 18:774-800

2. Voutilainen R, Franks S, Mason HD, Martikainen H. Expression of insulin-like growth factor (IGF), IGF binding protein, and IGF receptor messenger ribonucleic acids in normal and polycystic ovaries. J Clin Endocrinol Metab 1996;81:1003

3. Norman RJ, Davies MJ, Lord J, Moran LJ. The role of lifestyle modification in polycystic ovary syndrome. Trends Endocrinol Metab 2002;13:251-7

4. Pasquqli R, Fabbri R, Venturoli S, et al. Effect of weight loss and antiandrogenic therapy on sex hormone blood levels and insulin resistance in obese patients with polycystic ovaries. Am J Obstet Gynecol 1986;154(1):139-44 
5. Balen AH, Tan SL, Jacobs HS. Hypersecretion of luteinising hormone: a significant cause of infertility and miscarriage. Br J Obstet Gynaecol 1993;100:1082-9

6. Speroff L, Glass RH, Kase NG (Eds). Hirsutism; Gynecologic Endocrinology and Infertility, 6th Ed. William and Wilkins, Baltimore: 1999; pp. 523-56

7. Cela C, Robertson C, Rush K, et al. Prevalence of polycystic ovaries in women with androgenic alopecia. Eur J Endocrinol 2003;149:439-42

8. Schwartz RA. Acanthosis Nigricans. J Am Acad Dermatol 1994;31:1-19

9. Randle PJ, Garland PB, Hales CN, et al. The glucose fatty-acid cycle. Its role in insulin sensitivity and the metabolic disturbances of diabetes mellitus. Lancet $1963 ; 1: 785-9$

10. Dale PO, Tanbo T, Vaaler S, et al. Body weight, hyperinsulinaemia, and gonadotropin levels in the polycystic ovarian syndrome: evidence of two distinct populations. Fertil Steril 1992;58(3):487-91

11. Christian RC, Dumesic DA, Behrenbeck T, Oberg AL. Prevalence and predictors of coronary artery calcification in women with polycystic ovary syndrome. J Clin Endocrinol Metab 2003;88:2562-8

12. Dereli D, Ozgen G, Buyukkececi F, Guney E, et al. Platelet dysfunction in lean women with polycystic ovary syndrome and association with insulin sensitivity. J Clin Endocrinol Metab 2003;88:2263-8

13. Ovalle F, Azziz R. Insulin resistance, polycystic ovary syndrome and type 2 diabetes mellitus. Fertil Steril 2002;77:1095-1105
14. Holte J, Bergh T, Berne C, et al. Restored insulin sensitivity but persistently increased early insulin secretion after weight loss in obese women with polycystic ovary syndrome. J Clin Endocrinol Metab 1995;80(9):2586-93

15. Scalzo K. Case problem: dietary recommendations to combat obesity, insulin resistance, and other concerns related to polycystic ovary syndrome. J Am Diet Assoc 2000;100(8):955-7

16. Azziz R. The evaluation and management of hirsutism. Obstet Gynecol 2003;101:995-1007

17. Morin-Papunen LC, Vauhkonen I, Koivunen RM, et al. Endocrine and metabolic effects of metformin versus ethinyl estradiol-cypproterone acetate in obese women with polycystic ovary syndrome: a randomised study. J Clin Endocrinol Metab 2000;85:3161-8

18. Clark AM, Ledger W, Galletly C, et al. Weight loss results in significant improvement in pregnancy and ovulation rates in anovulatory obese women. Hum Reprod 1995;10:2705-12

19. Fedorcsak P, Dale PO, Storeng R, Abyholm T, Tanbo T. The effect of metformin on ovarian stimulation and in vitro fertilization in insulin-resistant women with polycystic ovary syndrome: an open-label randomized cross-over trial. Gynecol Endocrinol 2003;17(3):207-14

20. Kjotrod SB, von During V, Carlsen SM. Metformin treatment before IVF/ICSI in women with polycystic ovary syndrome; a prospective, randomized, double blind study. Hum Reprod 2004;19(6):1315-22 